SLAC-TN-10-040

LCLS-TN-08-3

\title{
Girder Alignment Plan
}

\author{
Zachary Wolf, Robert Ruland, Catherine LeCocq, \\ Eric Lundahl, Yurii Levashov, Ed Reese, Carl Rago, \\ Ben Poling, Donald Schafer, Heinz-Dieter Nuhn, Uli Wienands \\ SLAC
}

March 10, 2008

\begin{abstract}
The girders for the LCLS undulator system contain components which must be aligned with high accuracy relative to each other. The alignment is one of the last steps before the girders go into the tunnel, so the alignment must be done efficiently, on a tight schedule. This note documents the alignment plan which includes efficiency and high accuracy.
\end{abstract}

\section{Introduction $^{1}$}

The girders for the LCLS undulator system each support an undulator, undulator beam pipe, quadrupole, beam position monitor, and beam finder wire. These components must be aligned with respect to each other within tight tolerances. Additionally, the alignment must be made efficient since it is one of the last operations to be done and there will be considerable pressure to begin running the machine. This note presents the alignment plan, which incorporates efficiency and high accuracy.

The motivation for girder alignment involves the following considerations. Using beam based alignment, the girder position will be adjusted until the beam goes through the center of the quadrupole and beam finder wire. For the machine to work properly, the undulator axis must be on this line and the center of the undulator beam pipe must be on this line. The physics reasons for the undulator axis and undulator beam pipe axis to be centered on the beam are different, but the alignment tolerance for both are similar. In addition, the beam position monitor must be centered on the beam to preserve its calibration. Thus, the undulator, undulator beam pipe, quadrupole, beam finder wire, and beam position monitor axes must all be aligned to a common line. All relative alignments are equally important, not just, for example, between quadrupole and undulator. We begin by making the common axis the nominal beam axis in the girder coordinate system. All components will be initially aligned to this axis. A more accurate alignment will then position the components relative to each other, without incorporating the girder itself.

The alignment will be carried out in two steps: pre-alignment and alignment. A pre-alignment will be performed by standardizing components and using fixtures to position the components on the girders. The standardization will take place on various small coordinate measuring machines (CMMs) and will involve placing the axis of each component at a fixed location relative to the base of its mount. Fixtures will position the mounts of the components on the girder, aligning the axes of the components. Parallel efforts of CMM operators and crews using fixtures to assemble the girders will provide efficient pre-alignment. The bulk of the alignment work will be done in this step. All components will be positioned within $100 \mu \mathrm{m}$ of their ideal locations. When possible, better accuracy will be achieved.

\footnotetext{
${ }^{1}$ Work supported in part by the DOE Contract DE-AC02-76SF00515. This work was performed in support of the LCLS project at SLAC.
} 
Since the pre-alignment uses fixtures attached to the girder to position components, the girder itself must be built accurately. Each girder must be inspected before pre-alignment to ensure that the pre-alignment process will work. Similarly, the undulator beam pipes have limited horizontal adjustment. The undulator beam pipes must be inspected for horizontal straightness before prealignment.

After pre-alignment, the girder assemblies will be moved to a large coordinate measuring machine. This single machine will be used for final alignment of all the girders. The number of steps to be performed on this machine must be kept to a minimum so as not to become a bottleneck in the throughput. All components must come to this machine pre-aligned so that only small adjustments are necessary.

The details of the alignment step are determined by how the undulator and undulator beam pipe are mounted. The undulator's horizontal position can be easily changed, but changing its vertical position involves machining parts. Thus, the vertical position of the undulator can not be changed during alignment. The undulator beam pipe's vertical position can be easily changed, but changing its horizontal position involves starting the alignment over. The horizontal position of the undulator beam pipe can not be changed during alignment. The quadrupole and beam position monitor have mounts allowing easy horizontal and vertical motion. The beam finder wire is rigidly attached to the vacuum system and can not be moved vertically or horizontally during alignment using its mount. Internally it has provisions for adjustment, however, so its axis can also be aligned. The alignment involves horizontal movements of the undulator, quadrupole, beam position monitor, and beam finder wire card axis to the horizontal position of the axis of the undulator beam pipe. The alignment then involves vertical movements of the undulator beam pipe, quadrupole, beam position monitor, and beam finder wire card axis to the vertical position of the axis of the undulator. This is illustrated in figures 1 and 2 .
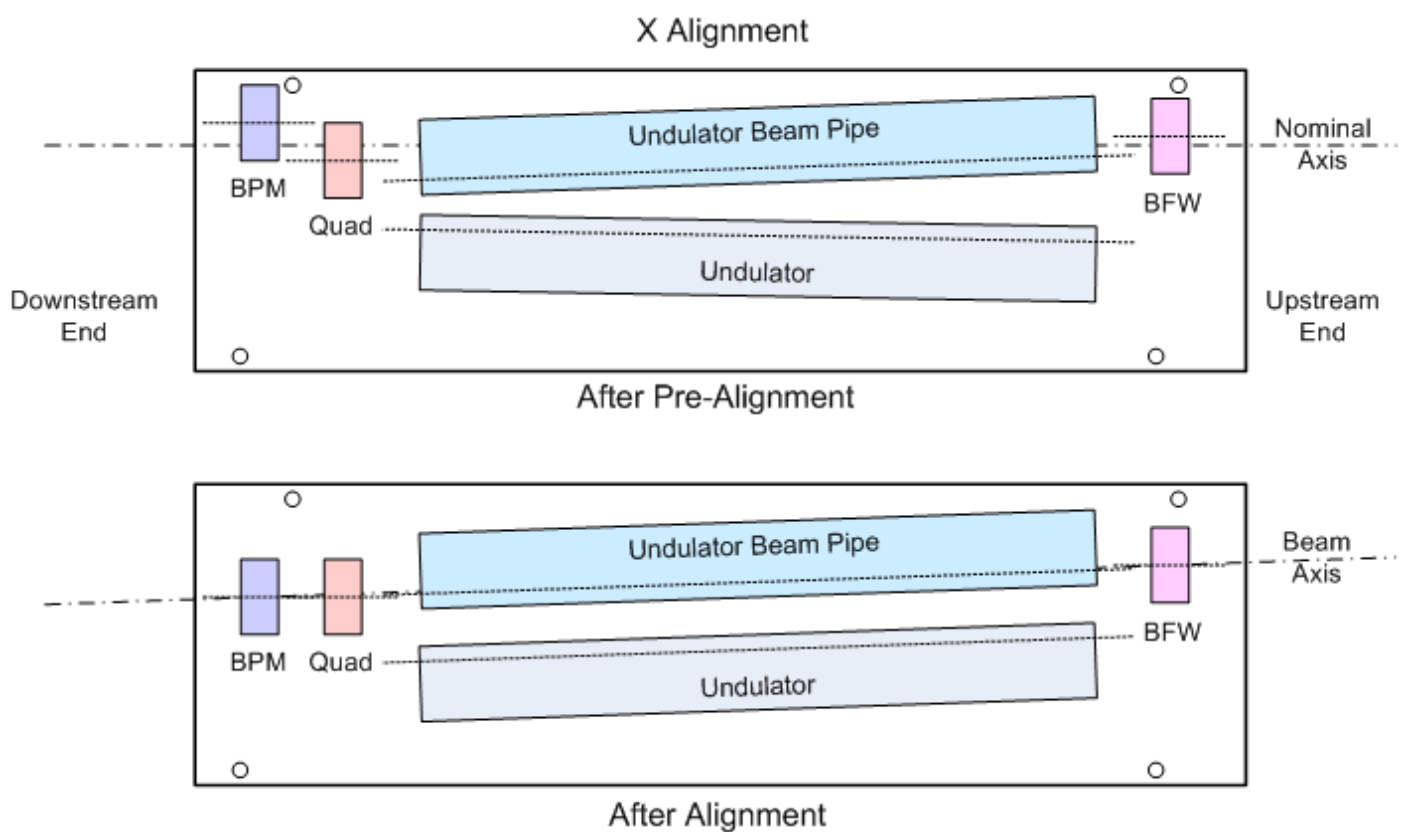

Figure 1: Components are aligned in $\mathrm{x}$ to the axis of the undulator beam pipe.

The note begins by giving an overview of the girder assembly. The components on the girder and the relevant alignment features are discussed. This is followed by the alignment accuracy 


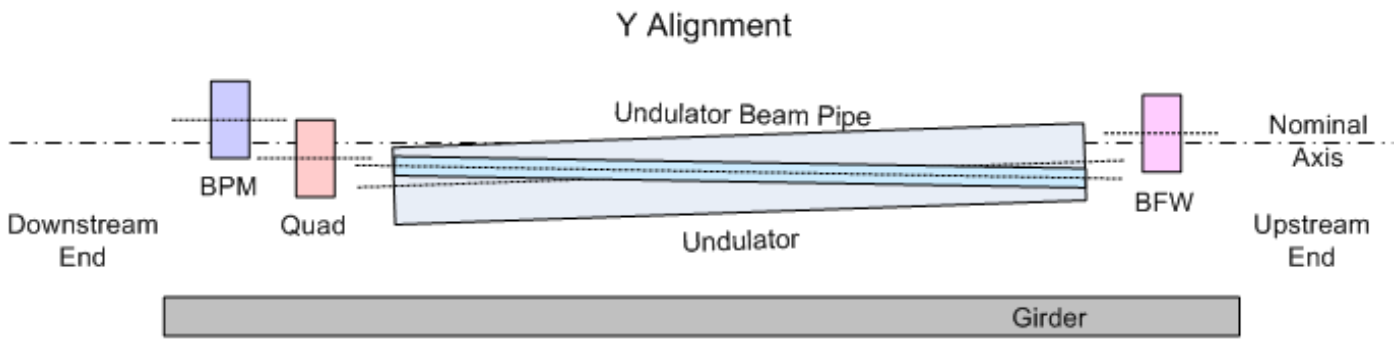

After Pre-Alignment

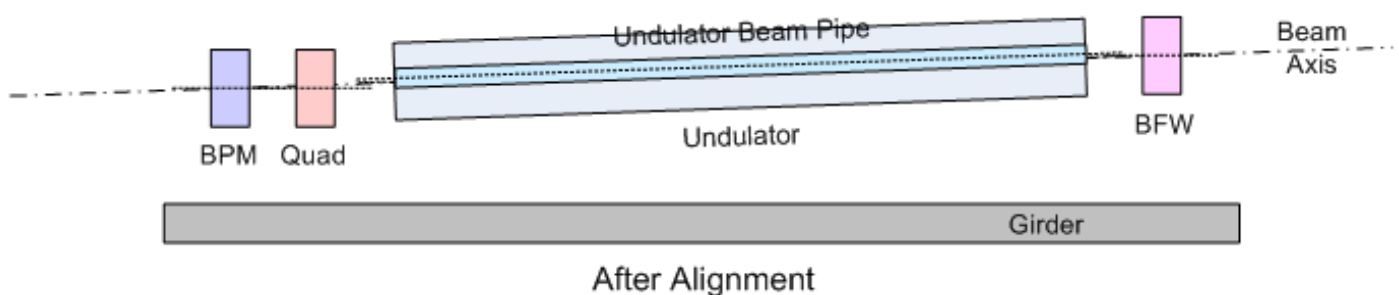

Figure 2: Components are aligned in y to the axis of the undulator.

requirements. The pre-alignment plan using standardization and fixturing and the alignment plan using the large CMM are then presented. The note concludes with a discussion of tests to perform to check the stability of the alignment.

\section{Girder Assembly Alignment}

\subsection{Overview}

The girder supports the undulator, undulator beam pipe, quadrupole, beam position monitor, and beam finder wire. A coordinate system is set up using features on the girder. All components are positioned to their desired locations or have their positions determined in this coordinate system. In this section of the note, the girder coordinate system is first described, followed by discussions of each component and the techniques used to align it.

\subsection{Girder Coordinate System}

The girder has four fiducial holes which are used to establish a coordinate system. The girder is described in an Engineering Specification Document ${ }^{2}$. The fiducial holes and component locations relative to the holes are documented in an engineering drawing ${ }^{3}$. The fiducial holes are illustrated in figure 3.

The holes are labeled U1 for the upstream hole near the beam pipe, U2 for the upstream hole on the back side of the girder, D1 for the downstream hole near the beam pipe, and D2 for the downstream hole on the back side of the girder. Each hole defines a unique point on the axis of the

\footnotetext{
${ }^{2}$ Jeff Collins et al., "LCLS Undulator Support/Mover System Engineering Specifications", LCLS Engineering Specifications Document 1.4-112.

${ }^{3}$ Drawing number L143-010000.
} 


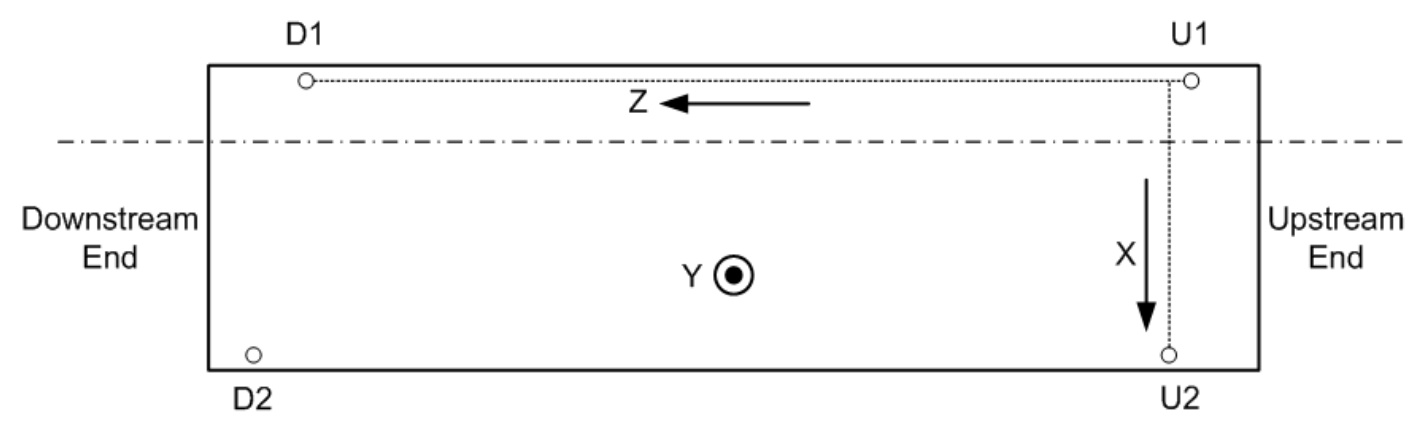

Figure 3: Girder fiducial holes are used to establish the girder coordinate system.

hole and flush with the surface. We use the same designations, U1, U2, D1, and D2, for the fiducial points defined by the holes.

The girder coordinate system is established as follows. A line drawn from U1 to D1 defines the direction of the $\mathrm{z}$ axis. A line perpendicular to the $\mathrm{z}$ axis through $\mathrm{U} 2$ defines the direction of the $\mathrm{x}$ axis. A line perpendicular to the $\mathrm{x}$ and $\mathrm{z}$ directions, away from the girder, defines the $\mathrm{y}$ direction. The origin of the coordinate system is U1. The coordinate system is uniquely defined.

The nominal beam axis is located at $x_{b}=173.95 \mathrm{~mm}$ and $y_{b}=258.67 \mathrm{~mm}$. The nominal undulator center is at $z_{u}=1717.04 \mathrm{~mm}$. The roll of the girder is established by making the $\mathrm{x}$ axis level with respect to gravity.

\subsection{Girder Reference Surfaces}

Components will be set to nominal positions in the pre-alignment step. Reference surfaces on the girder will be used to position the components. Fixtures will be bolted up against the reference surfaces and components will be positioned against the fixtures and bolted down. The reference surfaces are precision machined with fixed positions relative to the fiducialization holes. The reference surfaces are described in an Engineering Specification Document ${ }^{4}$ and are illustrated in figure 4 .

Downstream End

Upstream End

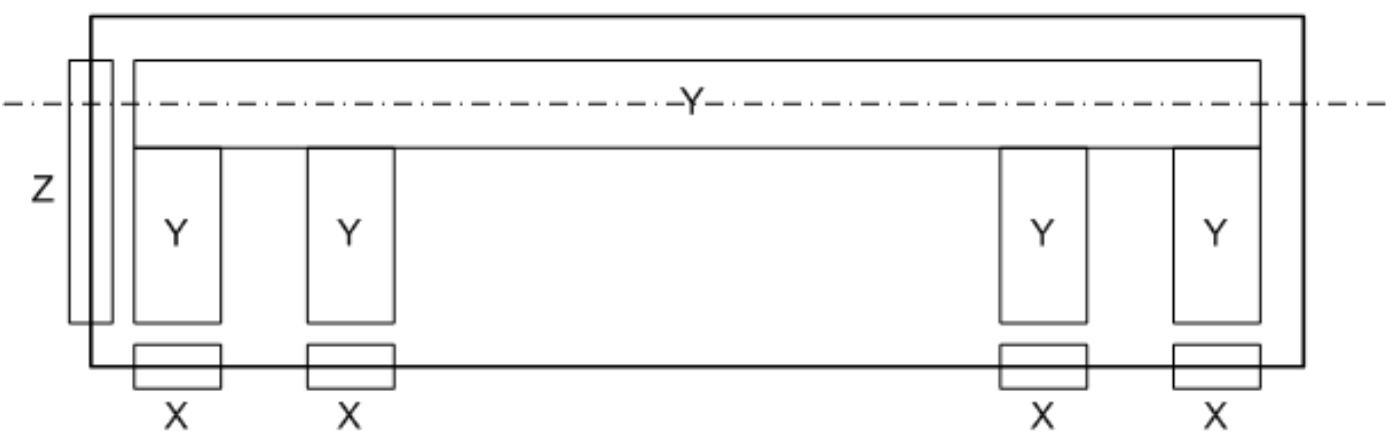

Figure 4: Reference surfaces on the girder are used to position components during pre-alignment.

\footnotetext{
${ }^{4}$ Jeff Collins et al., "LCLS Undulator Support/Mover System Engineering Specifications", LCLS Engineering Specifications Document 1.4-112.
} 
The long edge of the girder has four flats which will be used to set $\mathrm{x}$ and yaw coordinates during pre-alignment. The flats are marked $\mathrm{X}$ in the figure.

A large area on the top of the girder is machined flat and will be used to set $y$, pitch, and roll coordinates during pre-alignment. The machined flat is marked $\mathrm{Y}$ in the figure.

The entire downstream edge of the girder is a machined flat and will be used to set $\mathrm{z}$ and also yaw of components during pre-alignment. The flat is marked $\mathrm{Z}$ in the figure.

\subsection{Undulator}

The undulators are designed to be exchanged in the tunnel without additional alignment. To accomplish this, the ideal beam axis of each undulator is adjusted to be at the same location relative to its mount. The mount has registration surfaces which go against stops to define the undulator position. The ideal beam axis is determined magnetically and is known relative to the undulator tooling balls through a fiducialization process. In a standardization step, the ideal beam axis is set at a fixed location relative to the registration surfaces on the undulator mount.

The undulator sits on flat feet which are machined in order to position the undulator. This is illustrated in figure 5. All undulators are placed on the same reference girder and the feet are
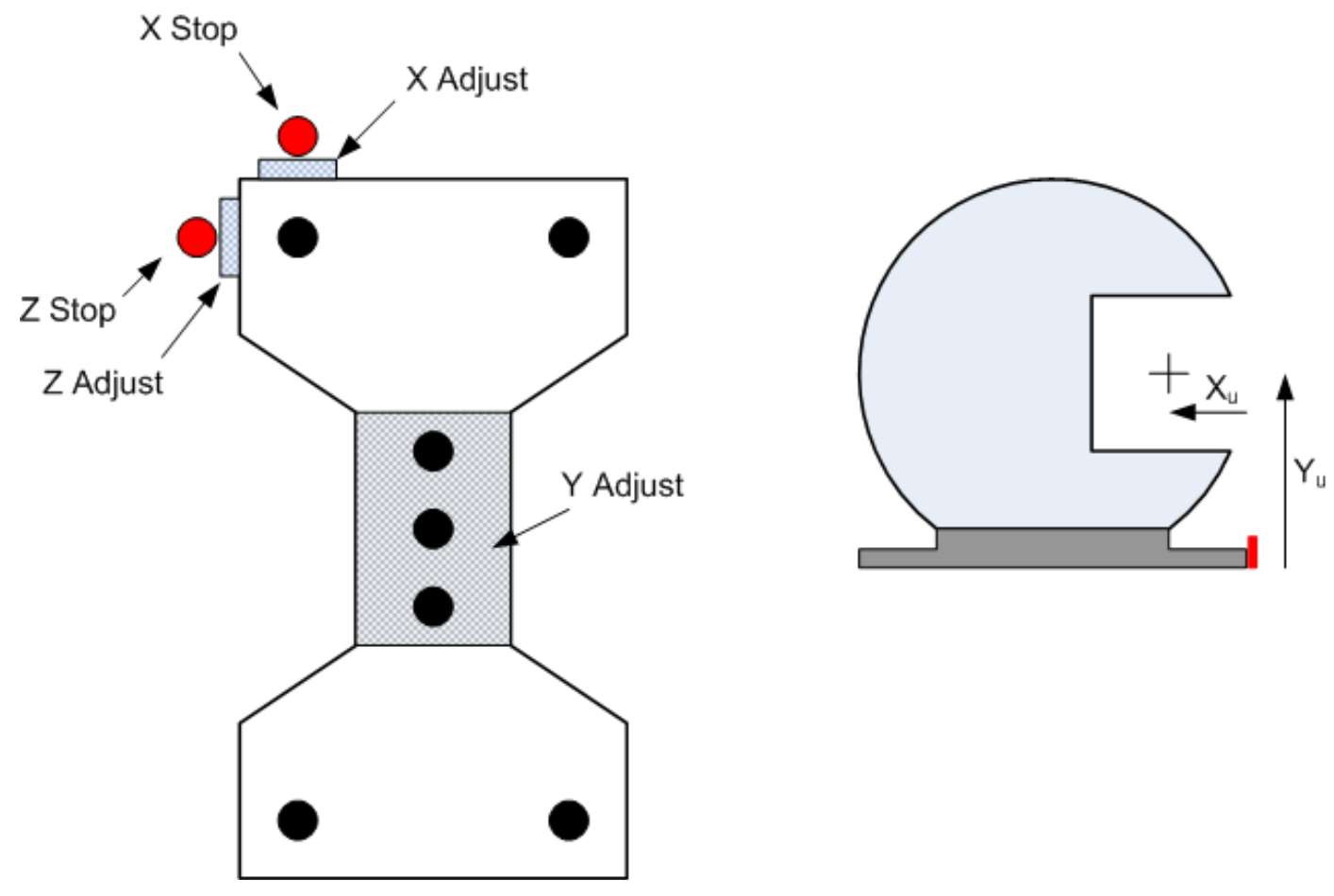

Figure 5: The undulator feet have pads which are machined in order to position the undulator. The pads are pushed against stops.

machined so the ideal beam axis is at the same (x,y) location for all undulators. The reference girder has a flat top and the y-adjust pads on the feet are machined to set the ideal beam axis at $y_{u}=164.54 \mathrm{~mm}$ above the flat surface and to take out any pitch or roll. The reference girder has two pins which position the undulator in $x$ and yaw. The x-adjust pads are machined so the ideal beam axis is $x_{u}=211.71 \mathrm{~mm}$ back from the pins. The reference girder also has a z-stop. A z-adjust pad is machined so the center of the undulator will be at $z=1717.04 \pm 0.50 \mathrm{~mm}$ in the 
girder coordinate system. The $(\mathrm{x}, \mathrm{y})$ location of the undulator on its mount is illustrated in figure 5 .

\subsection{Undulator Stages}

The undulator sits on two stages which set the position of the undulator. The stages have the $\mathrm{x}$-stop and z-stop described above attached to them. The top of each stage is a flat surface that the undulator foot sits on which sets the y position of the undulator. This is illustrated in figure 6 .

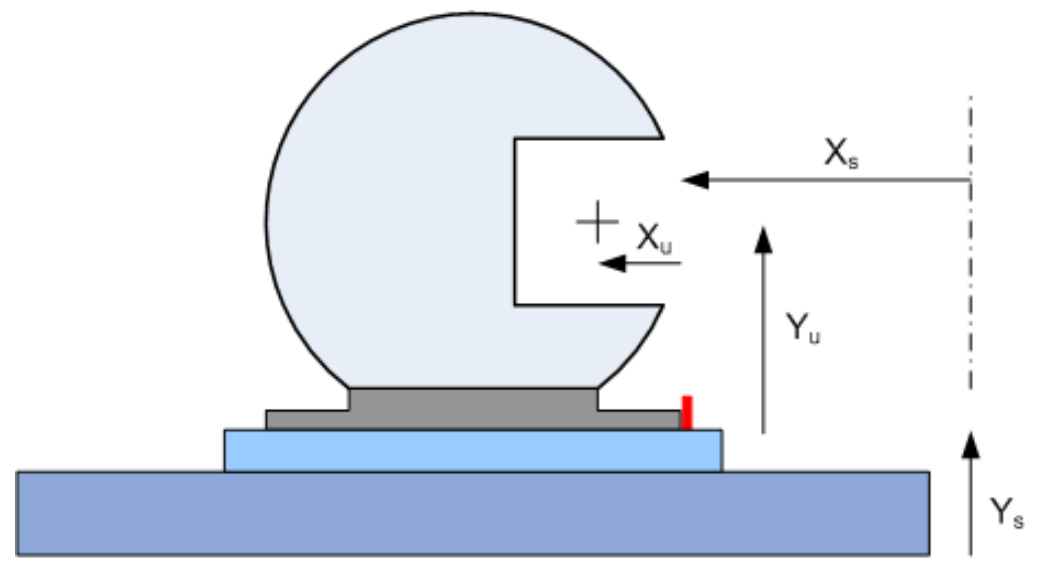

$(0,0)$

Figure 6: The undulator axis position in the girder coordinate system is determined by the stage position $\left(x_{s}, y_{s}\right)$ and the undulator axis position relative to the mount $\left(x_{u}, y_{u}\right)$.

In order for all undulators to be exchangeable, the stages must be adjusted so that a reference undulator sits with its ideal beam axis in the same position relative to the other components on each girder. For the reference undulator to sit in a unique relative position, several adjustments must be made. The stages must be set up to run parallel to each other. The top plate of the stages must be machined so that they are flat to the girder and the reference undulator sits at the proper height. The stages must be moved in $\mathrm{x}$ so the reference undulator's ideal beam axis is at the proper $\mathrm{x}$ location. A record of this $\mathrm{x}$ location must be made by recording the output of the linear potentiometers. The z-stop must be machined so the reference undulator has its center z position at the proper location.

Given the beam axis position in girder coordinates given above and the locations of the ideal beam axis relative to the undulator feet adjustment surfaces, the positions of the undulator feet stops can be determined in girder coordinates. In particular, the tops of the stages must be at $y_{s}=94.13 \mathrm{~mm}$.

\subsection{Undulator Beam Pipe}

The undulator beam pipe is an aluminum extrusion attached to a strongback. The beam pipe is approximately $3.46 \mathrm{~m}$ long. It is supported at 13 locations using a double nut system to set the height and roll. This is illustrated in figure 7 .

Horizontally, the beam pipe is stiff because of its large horizontal dimension. There are 4 adjustments on the mount to move the position of the chamber in $x$. The $y$ adjustments must be loosened to set $x$, however, so no straightening of the beam pipe in $x$ is possible using the mount. The global horizontal position will be set by pushing the beam pipe against a fixture on the girder 


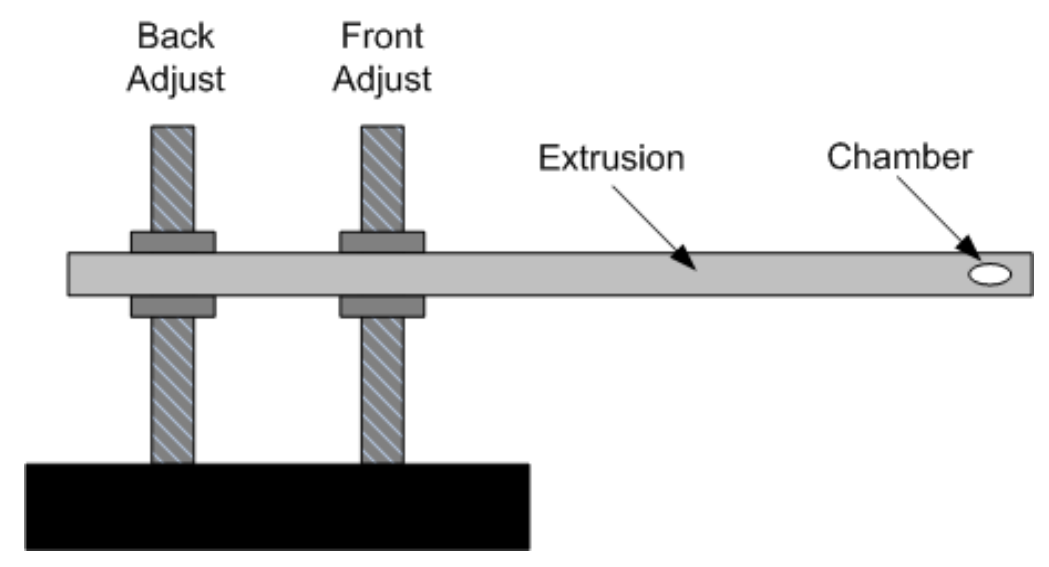

Figure 7: The undulator beam pipe is adjusted in height and roll using a double nut system.

and tightening bolts. The straightness of the beam pipe will be measured ahead of time and beam pipes with large bends will be repaired or returned.

Vertically, the beam pipe is flexible and requires adjustment at its 13 support locations. During the pre-alignment, both the front adjust and back adjust nuts will be used to set the center of the chamber to the correct height of $y_{b}=258.67 \mathrm{~mm}$ and also to take out any roll so that the extrusion does not contact the undulator. A series of indicators at the support locations will be used during the adjustments.

For the alignment on the CMM, both the top and bottom of the extrusion will be probed near the chamber. The average will be the estimate of the vertical position of the center of the chamber. The front adjust nuts will be used to set the center of the chamber to the correct height. The amount of roll introduced will be insignificant and will be ignored. The back adjust nuts will not be changed.

\subsection{Quadrupole}

The quadrupole is fiducialized using a vibrating wire measurement system. The system determines $x, y$, pitch, and yaw magnetically and $z$ and roll mechanically. The system specifies the ideal beam axis relative to tooling balls on the quadrupole. The quadrupole is aligned on the girder so that the ideal beam axis is along the girder beam axis.

The quadrupole mount is shown schematically in figure 8. Horizontal and vertical motions are provided. Roll, pitch, yaw, and $z$ motions are not provided.

The quadrupoles will be standardized on their mounts. The fiducialization data will be used to place the ideal beam axis at a fixed location relative to the bottom of the mount. A separate CMM will be used for this step. Both $x$ and $y$ will be adjusted using the mount. Roll, pitch, and yaw relative to the bottom of the mount will be corrected with shims if required.

Fixtures will be built for the pre-alignment to position the quadrupole mount at a fixed location relative to the girder. The quadrupole mount will be moved up against the fixture and bolted down. This will fix $x$, yaw, and $z$. The process will be fast and it is expected to position the quadrupole at the $100 \mu \mathrm{m}$ level.

During alignment, the tooling ball positions will be measured on the CMM. Small adjustments to $x$ and $y$ will be made. Roll, pitch, yaw, and $z$ will be checked to make sure they are within tolerance. 


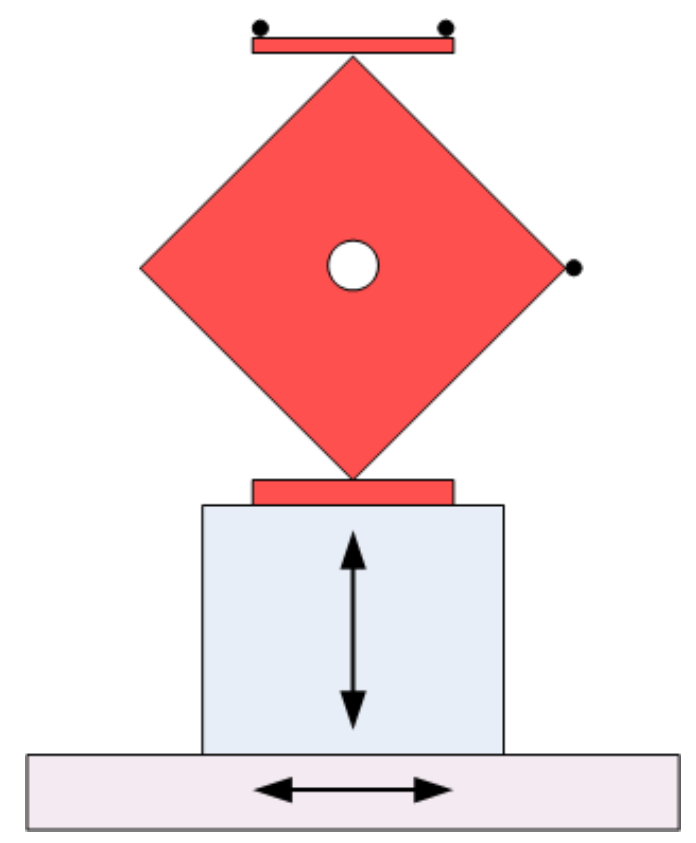

Figure 8: The quadrupole is mounted on two stages allowing horizontal and vertical movement.

\subsection{Beam Position Monitor}

The beam position monitor (BPM) is fiducialized mechanically. It is a precision machined piece whose outside surface accurately specifies its center position. It is aligned on the girder so that its mechanical center line is on the girder beam axis.

The BPM mount is shown schematically in figure 9. The mount provides six degree of freedom position adjustment. Three bolts position the BPM vertically and adjust pitch and roll. Two bolts position the BPM in $z$ and adjust yaw. One bolt moves the BPM in $x$. The adjustments are not simple, however, since they are coupled. The adjustment for pitch changes $z$, etc. Since the adjustments will be time consuming, the bulk of the adjustments should be done in the pre-alignment step.

The BPMs will be standardized on their mounts. A separate CMM will be used for this step. Roll, pitch, and yaw relative to the bottom of the mount will be corrected. The tolerances are fairly loose for these angles so further adjustment should not be required. Both $x$ and $y$ will be adjusted until the mechanical center of the BPM is at a fixed position relative to the mount.

Fixtures will be built for the pre-alignment to position the BPM mount at a fixed location relative to the girder. The BPM mount will be moved up against the fixture and bolted down. This will fix $x$, yaw, and $z . y$, pitch, and roll are set by the standardization and the flatness of the top of the girder. The process will be fast and it is expected to position the BPM at the $100 \mu \mathrm{m}$ level.

During alignment, the outside of the BPM will be measured on the CMM. Small adjustments to $x$ and $y$ will be made. Roll, pitch, yaw, and $z$ will be checked to make sure they are within tolerance.

\subsection{Beam Finder Wire}

The beam finder wire (BFW) is fiducialized mechanically. It consists of several components which are illustrated in figure 10. A card contains the wires for determining the $x$ and $y$ location of the 


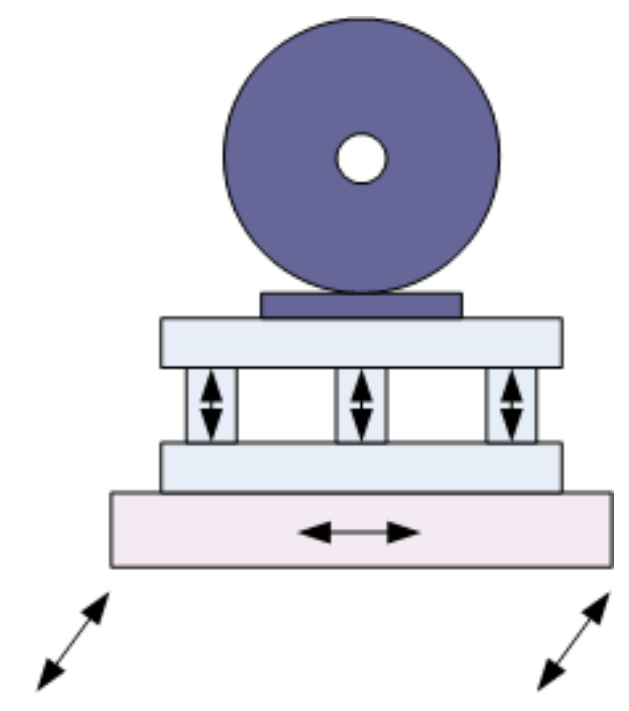

Figure 9: The beam position monitor mount provides six degree of freedom positioning.

beam. Fiducialization holes near the wires allow CMM probing without touching the wires. The fiducialization holes are related to the wire positions optically. Another aperture in the card allows the beam to pass unobstructed when the BFW is not in use. The card is attached to a movable frame driven by an actuator. The frame has balls attached to the top which register in stops which kinematically position the frame and wires. The stops are attached to the BFW housing through an adjustment mechanism. It allows moving the wire positions once the housing is in place. Tooling balls attached to the movable frame are used to determine the wire positions. Tooling balls are also attached to the housing for global alignment.

The ideal beam axis, the axis of the device, is offset from the wires. This is illustrated in figure 11. The axis is $0.4 \mathrm{~mm}$ from the vertical $\mathrm{x}$-wire in the negative $x$ direction. The axis is $0.2 \mathrm{~mm}$ from the horizontal y-wire in the positive $y$ direction.

The overall BFW assembly is attached to a six degree of freedom mount. Three bolts position the assembly vertically and adjust pitch and roll. Two bolts position the assembly in $z$ and adjust yaw. One bolt moves the assembly in $x$.

In a preliminary step, the wire positions are related optically to the fiducialization holes in the card. A small CMM determines the card position relative to the fixed tooling balls on the housing. Adjustments are made to the frame to place the wires at a fixed location relative to the tooling balls on the housing.

The BFWs will be standardized on their mounts. A separate CMM will be used for this step. Roll, pitch, and yaw relative to the bottom of the mount will be corrected. The tolerances are fairly loose for these angles so further adjustment should not be required. Both $x$ and $y$ will be adjusted until the wire positions of the $\mathrm{BFW}$ are at fixed positions relative to the mount.

Fixtures will be built for the pre-alignment to position the BFW mount at a fixed location relative to the girder. The BFW mount will be moved up against the fixture and bolted down. This will fix $x$, yaw, and $z$. $y$, pitch, and roll are set by the standardization and the flatness of the top of the girder. The process will be fast and it is expected to position the BFW at the $100 \mu \mathrm{m}$ level.

During alignment, the tooling balls on the BFW will be measured on the large CMM. Roll, pitch, yaw, and $z$ will be checked to make sure they are within tolerance. The tooling balls on the movable frame holding the wires will be measured to determine the wire positions, and therefore the axis position. The $x$ and $y$ position of the axis will be adjusted using the screws provided for 


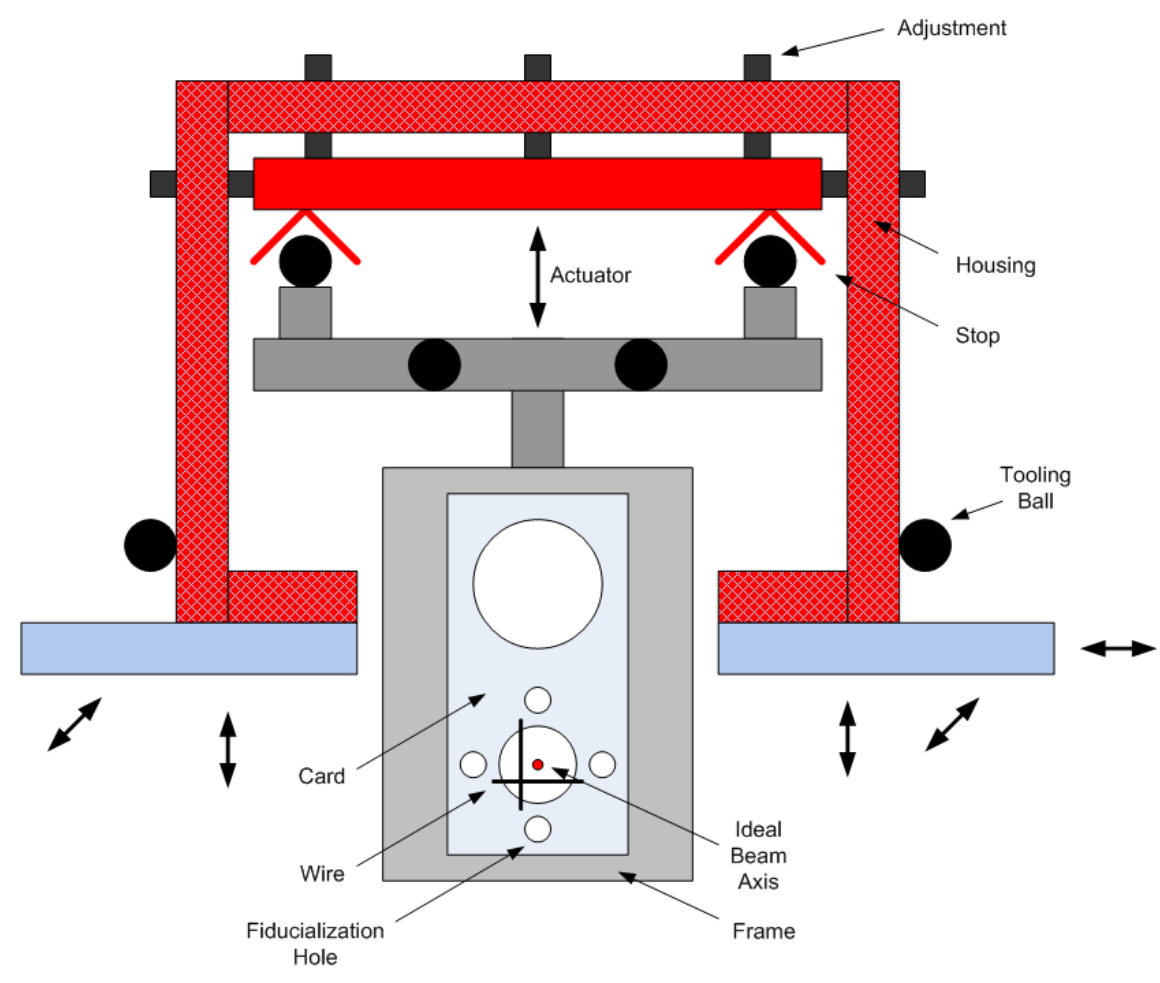

Figure 10: The beam finder wire position is initially adjusted using a six degree of freedom mount. Final alignment is done by moving the plate holding the stops which positions the movable wire card frame.

moving the kinematic mount.

\section{Requirements}

The girder alignment requirements come from documents referenced below. As noted previously, all components will be pre-aligned to their nominal positions. A final alignment will be made to the components using relative motions to optimize their alignment.

The requirements are made in an effort to align the undulator axis and the undulator beam pipe axis to a line drawn between the quadrupole center and the beam finder wire center to within 80 $\mu \mathrm{m}$ horizontally and $60 \mu \mathrm{m}$ vertically. Errors are divided equally, so each component is aligned to the nominal beam axis to $80 / \sqrt{2}=57 \mu \mathrm{m}$ horizontally and $60 / \sqrt{2}=42 \mu \mathrm{m}$ vertically. Relative errors between components are $80 \mu \mathrm{m}$ horizontally and $60 \mu \mathrm{m}$ vertically.

The requirements specify the accuracy with which each component must be aligned to the beam axis. For pre-alignment, the components are aligned to the nominal beam axis in the girder coordinate system, i.e. a line at $x_{b}=173.95 \mathrm{~mm}$ and $y_{b}=258.67 \mathrm{~mm}$. Components must be pre-aligned to the nominal beam axis within $100 \mu \mathrm{m}$.

For alignment, the beam axis is defined using the components. At the upstream end of the undulator, locate the point $\left(X_{u s}, Y_{u s}\right)$, where $X_{u s}$ is the $x$ position of the center of the undulator beam pipe chamber on the upstream end and $Y_{u s}$ is the $y$ position of the undulator on the upstream end. These values can not be adjusted, so this is a fixed point. At the downstream end of the undulator, locate the point $\left(X_{d s}, Y_{d s}\right)$, where $X_{d s}$ is the $x$ position of the center of the undulator 


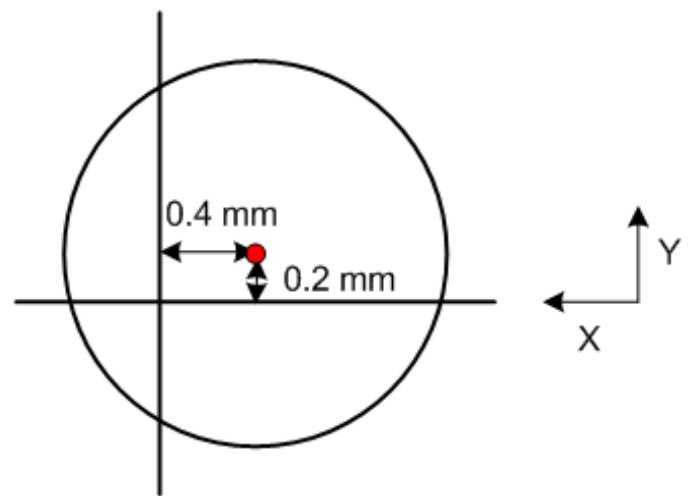

Figure 11: The BFW axis is offset from the wire positions.

beam pipe chamber on the downstream end and $Y_{d s}$ is the $y$ position of the undulator on the downstream end. The line between $\left(X_{u s}, Y_{u s}\right)$ and $\left(X_{d s}, Y_{d s}\right)$ defines the beam axis for the girder. All components will be aligned to the beam axis.

The list of alignment requirements follows.

\subsection{Undulator}

The undulator alignment requirements are given in a Physics Requirements Document ${ }^{5}$. The undulator is fiducialized such that the ideal beam axis position is known relative to tooling balls to $40 \mu \mathrm{m}$ vertically and $50 \mu \mathrm{m}$ horizontally. The undulator axis must be positioned relative to the quadrupole axis within $80 \mu \mathrm{m}$ horizontally and $60 \mu \mathrm{m}$ vertically; and similarly, the undulator axis must be positioned relative to the beam finder wire axis within $80 \mu \mathrm{m}$ horizontally and $60 \mu \mathrm{m}$ vertically. Assigning equal positioning errors to the undulator and other components, we arrive at the following requirements. Relative to the beam axis defined above:

1. The undulator axis must coincide with the beam axis within $\Delta x= \pm 0.06 \mathrm{~mm}$.

2. The undulator axis must coincide with the beam axis within $\Delta y= \pm 0.04 \mathrm{~mm}$.

3. The undulator center must be at $z=1717.04 \pm 0.50 \mathrm{~mm}$ in the girder coordinate system.

4. The undulator yaw must be below $35 \mu$ rad relative to the beam axis.

5. The undulator pitch must be below $24 \mu$ rad relative to the beam axis.

6. The undulator roll must be below $1 \mathrm{mrad}$ in the girder coordinate system.

\subsection{Undulator Stages}

The undulator stages must meet the following requirements ${ }^{6}$ :

1. The translation stages must be parallel to $\pm 12.5 \mu \mathrm{m}$ over their travel. The primary requirement is that they do not bind when in use.

\footnotetext{
${ }^{5}$ H. D. Nuhn et al., "General Undulator System Requirements", LCLS Physics Requirements Document 1.4-001.

${ }^{6}$ Jeff Collins et al., "LCLS Undulator Support/Mover System Engineering Specifications", LCLS Engineering Specifications Document 1.4-112.
} 
2. The translation stages must have their top mounting surfaces at the same height to $\pm 12.5 \mu \mathrm{m}$ over the area under the weight bearing section of the undulator feet.

3. The center of the top surfaces of the translation stages must be at a nominal height of $y_{s}=$ $94.13 \pm 0.1 \mathrm{~mm}$ in the girder coordinate system. Other components will be set to the actual undulator height.

\subsection{Undulator Beam Pipe}

The undulator beam pipe must meet the following alignment requirements ${ }^{7,8}$ :

1. The beam pipe chamber axis must be adjusted straight to within $\pm 200 \mu \mathrm{m}$.

2. A fitted line giving the beam pipe chamber axis must coincide with the beam axis within $\Delta x= \pm 0.06 \mathrm{~mm}$.

3. A fitted line giving the beam pipe chamber axis must coincide with the beam axis within $\Delta y= \pm 0.04 \mathrm{~mm}$.

4. The average beam pipe chamber center must be at $z=1717.04 \pm 0.50 \mathrm{~mm}$ in the girder coordinate system.

5. A fitted line giving the beam pipe chamber axis must have yaw below $35 \mu$ rad relative to the beam axis.

6. A fitted line giving the beam pipe chamber axis must have pitch below $24 \mu \mathrm{rad}$ relative to the beam axis.

7. The beam pipe roll must be below $1 \mathrm{mrad}$ in the girder coordinate system to provide clearance for the undulator. The worst case $2 \mathrm{mrad}$ roll relative to the undulator uses up $0.1 \mathrm{~mm}$ of clearance when the beam pipe is fully inserted.

\subsection{Quadrupole}

The quadrupole alignment must meet the following requirements ${ }^{9}$ :

1. The quadrupole axis must coincide with the beam axis within $\Delta x= \pm 0.06 \mathrm{~mm}$.

2. The quadrupole axis must coincide with the beam axis within $\Delta y= \pm 0.04 \mathrm{~mm}$.

3. The quadrupole center must be at $z=3524.33 \pm 0.50 \mathrm{~mm}$ in the girder coordinate system.

4. The quadrupole yaw must be below $10 \mathrm{mrad}$ relative to the beam axis.

5. The quadrupole pitch must be below $10 \mathrm{mrad}$ relative to the beam axis.

6. The quadrupole roll must be below $5 \mathrm{mrad}$ in the girder coordinate system.

\footnotetext{
${ }^{7}$ S. H. Lee et al., "Undulator Vacuum Chamber Requirements", LCLS Engineering Specification Document 1.4-118. ${ }^{8}$ H. D. Nuhn et al., "General Undulator System Requirements", LCLS Physics Requirements Document 1.4-001.

${ }^{9}$ H. D. Nuhn et al., "General Undulator System Requirements", LCLS Physics Requirements Document 1.4-001.
} 


\subsection{Beam Position Monitor}

The BPM alignment must meet the following requirements ${ }^{10}$ :

1. The BPM axis must coincide with the beam axis within $\Delta x= \pm 0.06 \mathrm{~mm}$.

2. The BPM axis must coincide with the beam axis within $\Delta y= \pm 0.04 \mathrm{~mm}$.

3. The BPM center must be at $z=3655.61 \pm 0.50 \mathrm{~mm}$ in the girder coordinate system.

4. The BPM yaw must be below $10 \mathrm{mrad}$ relative to the beam axis.

5. The BPM pitch must be below $10 \mathrm{mrad}$ relative to the beam axis.

6. The BPM roll must be below 5 mrad in the girder coordinate system.

\subsection{Beam Finder Wire}

The BFW alignment must meet the following requirements ${ }^{11}$ :

1. The BFW axis must coincide with the beam axis within $\Delta x= \pm 0.06 \mathrm{~mm}$.

2. The BFW axis must coincide with the beam axis within $\Delta y= \pm 0.04 \mathrm{~mm}$.

3. The BFW center must be at $z=-77.77 \pm 0.50 \mathrm{~mm}$ in the girder coordinate system.

4. The BFW housing yaw must be below $10 \mathrm{mrad}$ in the girder coordinate system.

5. The BFW housing pitch must be below $10 \mathrm{mrad}$ in the girder coordinate system.

6. The BFW housing roll must be below 5 mrad in the girder coordinate system.

\section{Pre-Alignment Plan}

\subsection{Undulator Stages}

Once the undulator stages are attached to the girder and the girder coordinate system is established, the stage positions must be checked and possibly adjusted. The stages are positioned on the girder by placing the stages against precision pins and bolting them down.

1. Visually check that the stages are positioned against their pins. This only checks the positions of the outer frame of the stages and is not a guarantee that the travel or top plate positions on the stages meet specifications. Loosen the bolts and adjust the stages if necessary.

2. Check that the stages run parallel to each other over their travel. Bolt an object which simulates an undulator to the stages. Run the stages back and forth. Verify that the stages do not bind. If they do, adjust the stages so they are parallel and run smoothly.

3. Using height gauges on the girder, check the height of the top surfaces of the plates on the undulator stages relative to the girder. If they are not at $y=94.132 \pm 0.1 \mathrm{~mm}$ in the girder coordinate system, flag the girder as needing repair. CMM measurements will be required. This is a coarse check only to allow the reference undulator to slip over the beam pipe on the CMM. The stage heights will be measured on the CMM. If they are out of tolerance, they will need to be removed and the bottom surfaces machined to bring them into tolerance.

\footnotetext{
${ }^{10}$ H. D. Nuhn et al., "General Undulator System Requirements", LCLS Physics Requirements Document 1.4-001.

${ }^{11}$ H. D. Nuhn et al., "General Undulator System Requirements", LCLS Physics Requirements Document 1.4-001.
} 
4. Use alignment fixtures to set $\mathrm{x}, \mathrm{z}$, and yaw of the plates on top of the undulator stages. Exact locations in $\mathrm{x}$ and $\mathrm{z}$ of these plates is not critical, however, fixturing must be used to set them so that bolt hole patterns will be in the proper locations for undulator mounting.

\subsection{Undulator Beam Pipe}

1. Inspect the beam pipe using a CMM. Verify that the beam pipe is straight in $x$ to $\pm 0.2 \mathrm{~mm}$. If this is not the case, return the beam pipe for straightening or repair.

2. Attach the beam pipe support structure to the girder. Attach the beam pipe to the support structure. Attach the beam pipe horizontal positioning fixture to the girder.

3. Loosen the bolts attaching the vertical adjustment to the support. Slide the vertical adjustment and beam pipe assembly horizontally so that the beam pipe touches the fixture. This will position the beam pipe at $x=173.95 \pm 0.1 \mathrm{~mm}$ and set the yaw below $60 \mu \mathrm{rad}$. Simultaneously, move the assembly so the beam pipe center is at $z=1717.04 \pm 0.50 \mathrm{~mm}$ on the girder. Tighten the bolts.

4. Remove the beam pipe and support assembly from the girder and place it on a surface plate.

5. Using the surface plate and height gauges, check the height of the beam pipe along its length. Using the adjustment bolts, set the height of the center of the chamber to $y=258.67 \pm 0.1$ $\mathrm{mm}$ above the surface plate. Simultaneously, use the double adjustment bolts to reduce the roll of the beam pipe to below $1 \mathrm{mrad}$. This straightens the beam pipe vertically and is a coarse adjustment of the average height allowing the undulator to move past the beam pipe on the CMM. A final straightening and setting of the average height will take place on the CMM.

6. Place the beam pipe and its support structure on the girder. Pins position the support structure on the girder, there is no adjustment. Tighten all bolts to rigidly attach the beam pipe assembly to the girder.

\subsection{Quadrupole}

The quadrupole pre-alignment is performed in two steps. First, all quadrupoles are standardized so that the magnetic center is at a fixed location relative to the quadrupole mount. Then, the mounts are placed against fixtures to position the quadrupole on the girder.

1. Build two fixtures, one to position the quadrupole on the girder and the other to position the quadrupole on the CMM.

2. Standardize all quadrupoles. Place the quadrupole against the fixture on the CMM. Check whether the yaw, pitch, or roll is out of tolerance. If so, shim the quadrupole to be within tolerance. Using the adjustments in the mount, adjust $x$ and $y$ of the quadrupole center to the same value for all quadrupoles. Set the $y$ value to $y=258.67 \pm 0.1 \mathrm{~mm}$. The $x$ value depends of the details of the fixturing, however, it must be set so that when the quadrupole is placed against the fixture on the girder, the center of the quadrupole is at $x=173.95 \pm 0.1$ $\mathrm{mm}$.

On the girder:

1. Attach the quadrupole positioning fixture to the girder. This fixture sets $x$, yaw, and $z$.

2. Push the quadrupole mount up against the positioning fixture.

3. Bolt the quadrupole mount to the girder. 


\subsection{Beam Position Monitor}

The BPM pre-alignment is performed in two steps. First, all BPMs are standardized so that the device center is at a fixed location relative to the mount. Then, the mounts are placed against fixtures to position the BPM on the girder.

1. Build two fixtures, one to position the BPM on the girder and the other to position the BPM on the CMM.

2. Standardize all BPMs. Place the BPM against the fixture on the CMM. Check whether the yaw, pitch, or roll is out of tolerance. If so, using the mount, adjust the BPM to be within tolerance. Using the adjustments in the mount, adjust $x$ and $y$ of the BPM center to the same value for all BPMs. Set the $y$ value to $y=258.67 \pm 0.1 \mathrm{~mm}$. The $x$ value depends of the details of the fixturing, however, it must be set so that when the BPM is placed against the fixture on the girder, the center of the BPM is at $x=173.95 \pm 0.1 \mathrm{~mm}$.

On the girder:

1. Attach the BPM positioning fixture to the girder. This fixture sets $x$, yaw, and $z$.

2. Push the BPM mount up against the positioning fixture.

3. Bolt the BPM mount to the girder.

\subsection{Beam Finder Wire}

The BFW pre-alignment is performed in three steps. First, the wire positions are standardized relative to the tooling balls on the housing. Second, the tooling balls on the housing are standardized so that they are at a fixed location relative to the mount. Third, the mounts are placed against fixtures to position the BFW on the girder.

Position the wires:

1. Optically locate the wire positions relative to the fiducialization holes on the wire cards.

2. Using a CMM, locate the holes on the wire cards relative to the tooling balls on the movable frame holding the wire card and also relative to the tooling balls on the housing of the BFW.

3. Adjust the frame holding the wire card until the BFW axis is at a fixed location relative to the tooling balls on the housing. The coordinates of the axis relative to the tooling balls is not critical. It must, however, be recorded and be the same for all assemblies.

Standardize the assemblies:

1. Build two fixtures, one to position the BFW on the girder and the other to position the BFW on the CMM.

2. Standardize all BFWs. Place the BFW against the fixture on the CMM. Check whether the yaw, pitch, or roll is out of tolerance. If so, using the mount, adjust the BFW to be within tolerance. Using the adjustments in the mount, adjust $x$ and $y$ of the BFW axis to the same value for all BFWs. Set the $y$ value to $y=258.67 \pm 0.1 \mathrm{~mm}$. The $x$ value depends of the details of the fixturing, however, it must be set so that when the BFW is placed against the fixture on the girder, the axis of the $\mathrm{BFW}$ is at $x=173.95 \pm 0.1 \mathrm{~mm}$.

On the girder:

1. Attach the BFW positioning fixture to the girder. This fixture sets $x$, yaw, and $z$.

2. Push the BFW mount up against the positioning fixture.

3. Bolt the BFW mount to the girder. 


\section{Alignment Plan}

Before alignment, let the girder sit in the CMM room for several days to stabilize its temperature at $20{ }^{\circ} \mathrm{C}$. All measurements done on the CMM must have the girder supported by a kinematic mount, the same way it will be mounted in the tunnel. Place the reference undulator on the girder after it is placed on the CMM.

\subsection{Initial Position Measurements}

Alignment involves small motions of the components relative to each other in order to put their axes on a single line. For practical reasons, this single line, the beam axis, is not in a fixed location relative to the girder. The girder is used, however, to set up the coordinate system in which position measurements are made. In order to start the alignment, the starting positions of the components must be known. The horizontal position of the undulator beam pipe axis and the vertical position of the undulator axis will be the positions all other components are moved to.

1. Establish a coordinate system. Use the CMM to find the locations of the fiducialization holes in the girder. Set up the girder coordinate system as previously described.

2. Using the CMM, measure the positions of all components on the girder. Verify that the pre-alignment was successful and only small adjustments to the components are necessary.

3. Measure the horizontal position of the undulator beam pipe axis at a number of points along its length. Fit a line to the points. On the upstream end, calculate from the fit $X_{u s}$, the $x$ position of the undulator beam pipe axis at the upstream end of the beam pipe. On the downstream end, calculate from the fit $X_{d s}$, the $x$ position of the undulator beam pipe axis at the downstream end of the beam pipe. All other components will be adjusted in $x$ to $X_{u s}$ and $X_{d s}$. The pre-alignment will limit yaw errors so that further corrections for actual $z$ positions of components will be ignored.

4. From the undulator fiducialization data and the undulator tooling ball position measurements, determine the $y$ position of the undulator axis as a function of $z$. In particular, determine $Y_{u s}$ and $Y_{d s}$, the upstream and downstream $y$ positions of the undulator axis at the ends of the undulator. All other components will be adjusted in $y$ to $Y_{u s}$ and $Y_{d s}$. The pre-alignment will limit pitch errors so that further corrections for actual $z$ positions of components will be ignored.

\subsection{Undulator Beam Pipe}

1. Fit a line to the measured $x$ positions of the beam pipe at a number of $z$ locations taken in the initial position measurements. Verify that the rms deviation from the line (straightness) is less than $0.2 \mathrm{~mm}$. If it is not, consult the physicist in charge about whether to have a new beam pipe installed.

2. Using the CMM, determine the $z$ positions of the faces of the flanges on both ends of the beam pipe. Determine the $z$ position of the center of the beam pipe. Verify that the position is $z=1717.04 \pm 0.50 \mathrm{~mm}$. The main criterion is that the vacuum system bolts up. The $z$ position should only be recorded.

3. Using the CMM, measure the $y$ positions on the upper surface and lower surface of the beam pipe at many $z$ locations. Average the $y$ values from the upper and lower surface at each $z$ location. The average is the estimate of the $y$ position of the center of the chamber. Fit the average $y$ positions as a function of $z$. Use the fit to determine $y$, pitch, and rms straightness 
of the beam pipe. If the fit does not go through $Y_{u s} \pm 0.04 \mathrm{~mm}$ on the upstream end and $Y_{d s} \pm 0.04 \mathrm{~mm}$ on the downstream end, use the front adjustment bolts to align the beam pipe along its length to the undulator axis. If the chamber is not straight to within $\pm 200 \mu \mathrm{m}$, correct the problem using the front adjustment bolts. The effect on roll of using only the front adjustment bolts should be minimal because of the pre-alignment. Repeat this step if any adjustments are made.

\subsection{Undulator}

1. Determine the $\mathrm{z}$ location of the center of the reference undulator. Have the z-stop on the undulator stage machined until the center of the undulator is within $0.5 \mathrm{~mm}$ of its nominal location.

2. Determine the $\mathrm{x}$ position and yaw of the axis of the reference undulator. Remove the reference undulator and move the stages by running their motors in order to place the undulator axis at $x=X_{u s} \pm 0.06 \mathrm{~mm}$ on the upstream end and $x=X_{d s} \pm 0.06 \mathrm{~mm}$ on the downstream end. Replace the undulator and check the position of the ideal beam axis. Repeat until the ideal beam axis is in the proper location within $0.06 \mathrm{~mm}$. Record the linear potentiometer position values on each stage. The undulator stages must be returned to these linear potentiometer position values when in use.

\subsection{Quadrupole}

1. Using the CMM, locate all tooling balls on the quadrupole. From the fiducialization data, determine the $x, y, z$, roll, pitch, and yaw coordinates of the quadrupole axis.

2. If roll, pitch, or yaw is out of tolerance, shim the quadrupole.

3. If the $z$ position is out of tolerance, loosen the bolts attaching the quadrupole mount to the girder. Move the quadrupole center to $z=3524.33 \pm 0.50 \mathrm{~mm}$. Re-tighten the bolts.

4. Using the adjustment in the mount, set the quadrupole center to $x=X_{d s} \pm 0.06 \mathrm{~mm}$.

5. Using the adjustment in the mount, set the quadrupole center to $y=Y_{d s} \pm 0.04 \mathrm{~mm}$.

6. If any adjustments were made, repeat these steps.

\subsection{Beam Position Monitor}

1. Using the CMM, locate the mechanical axis of the BPM. Determine the $x, y, z$, roll, pitch, and yaw coordinates of the BPM axis.

2. If roll, pitch, or yaw is out of tolerance, adjust the BPM.

3. Because of vacuum system constraints, the $z$ position can only be recorded.

4. Using the adjustment in the mount, set the BPM center to $x=X_{d s} \pm 0.06 \mathrm{~mm}$.

5. Using the adjustment in the mount, set the BPM center to $y=Y_{d s} \pm 0.04 \mathrm{~mm}$.

6. If any adjustments were made, repeat these steps. 


\subsection{Beam Finder Wire}

1. Using the CMM, locate all tooling balls on the BFW. From the fiducialization data, determine the $x, y, z$, roll, pitch, and yaw coordinates of the BFW axis.

2. Because of vacuum system constraints, no adjustments to the BFW housing position can be made using the mount.

3. Using the adjustment in the kinematic stop of the movable frame, set the BFW axis to $x=$ $X_{u s} \pm 0.06 \mathrm{~mm}$.

4. Using the adjustment in the kinematic stop of the movable frame, set the BFW axis to $y=$ $Y_{u s} \pm 0.04 \mathrm{~mm}$.

5. If any adjustments were made, repeat these steps.

\subsection{Final Position Measurements}

After all adjustments have been made, use the CMM to locate all components. Set up a coordinate system using the girder fiducial holes. All tooling ball locations on the undulator, quadrupole, and BFW are to be measured. Mechanical measurements of the outside of the BPM are to be made. Scans along the undulator beam pipe giving its $x$ and $y$ location must be performed. Run analysis software which incorporates fiducialization data to verify that the axis of each component is properly located.

\section{Tests}

The girder is a large, heavy object with different materials which must keep its precision alignment. A number of tests are necessary to verify the girder alignment.

\subsection{Pre-Alignment Check}

After the first few girders are pre-aligned, survey crews will check the positions of all the components. Fixturing will be adjusted to make sure each component is aligned to $100 \mu \mathrm{m}$. Afterward, CMM measurements will be used as feedback to further fine tune the fixturing, if necessary.

\subsection{Undulator Position Repeatability Test}

On the CMM, place the undulator on the stages. Record the tooling ball positions. Remove the undulator and replace it. Again record the tooling ball position. Verify that the undulator position is repeatable within tolerance.

\subsection{Undulator Roll Out Test}

Place the girder on the CMM on a kinematic mount. Place an undulator on the girder. Record the undulator, quadrupole, and BFW tooling ball positions. Move the undulator to the roll out position. Record all tooling ball values. Move the undulator back to its home position. Record all tooling ball values. Note the motion of the quadrupole and BFW in the roll out position. Note the repeatability of all component positions. 


\subsection{Girder Handling Test}

Place a girder on the CMM. Measure all component positions. Handle the girder using the proposed methods. This involves packaging the girder, moving the girder from the MMF to storage, and from storage to the tunnel. Bring the girder back to the CMM and repeat the measurements of all component positions. Verify that the girder handling techniques to not change the alignment of the girder.

\subsection{Girder Temperature Test}

Place the girder on the CMM. Measure all component positions. Take the girder to a location where the temperature can be varied. Adjust the temperature to $15{ }^{\circ} \mathrm{C}$. Let the girder remain for two days. Afterward, bring the girder back to the CMM and measure the positions of all the components. Verify that the girder alignment can withstand $-5{ }^{\circ} \mathrm{C}$ temperature excursions. Repeat this test changing the girder temperature to $25^{\circ} \mathrm{C}$. 